\title{
Dynamic and Static Measures of Growth Among Pre- and Postmenarcheal Females in Rural Bangladesh
}

\author{
ANN P. RILEY \\ Population Studies Center, The University of Michigan, Ann Arbor, \\ Michigan 48104-2590
}

\begin{abstract}
Height and weight for age and height and weight velocity are examined in a sample of Bangladeshi adolescents aged 10-20 years with an average age at menarche of 15.8 years. Interpopulation differences between preand postmenarcheal girls are assessed and age patterns are compared to standards of U.S. and British adolescents. Bangladeshi adolescents are shorter and lighter for their age and lighter for their height than are U.S. adolescents. The growth spurt in height and weight is delayed and spread out over time, and growth rates are lower throughout the spurt when compared to British and U.S. girls. As in developed countries, most girls attain menarche after peak height velocity, while weight gain per year is highest around the time of menarche. There is no significant relationship between age at menarche and height or weight at menarche.
\end{abstract}

The timing of age at menarche and adolescent growth in chronically malnourished populations may have important implications for reproductive health, particularly in social settings where menarche is an important marker of readiness for marriage. The effect of young maternal age on first birth has emerged as a central concern in developed and developing countries, and the relative importance of biological factors attributed to immaturity of the mother visà-vis socioeconomic factors has not been fully resolved.

Some important questions remain unanswered regarding the long-term effects of malnutrition on birth outcome and maternal health. Is the adolescent growth spurt in undernourished adolescents similar to that of well-nourished girls in shape, but moved forward in time; or is the process somewhat different in other characteristics besides the starting and ending points? How mature are late adolescents and young adult women when they first conceive? Is it plausible that young women may conceive before their growth spurt in height and weight is complete, which might compromise birth outcome or their own health?

In the present study growth in height and weight are described in relation to chronological age and menarcheal status in a sample of adolescent females in rural Bangladesh. The population of Bangladesh suf- fers from chronic malnutrition of varying degrees, and is plagued by periodic manmade and natural disasters that result in acute food shortages. The analysis presented here is descriptive in nature and examines differences in nutritional status (e.g., height and weight) by age group and by menarcheal status. These measures are compared to studies of well-nourished adolescents.

These data provide detailed anthropometric information on a population of adolescents where chronic malnutrition is widespread, age at menarche is delayed, and marriage and childbearing begin at a relatively early age. (The average age at menarche in Matlab is 15.8 years, and the average interval from menarche to conception is 1.5 years [see Foster et al., 1986].) Similar measures of growth are not available elsewhere on Bangladeshi adolescents, to the author's best knowledge. These data also contain a longitudinal component that allows rates of growth in height and weight by age and by menarche status to be studied, as well as static measures of height and weight.

\section{BACKGROUND}

The onset and timing of adolescent growth and development are highly variable among

Received May 4, 1989; accepted January 10, 1990. 
and within populations (Tanner, 1978; Eveleth and Tanner, 1976; Wyshak, 1983). On average, the onset of the adolescent growth spurt occurs between 10.5 and 13 years in European girls (Frisch, 1972; Marshall and Tanner, 1979). Chronic undernutrition slows height velocity during childhood and adolescence and prolongs the period of growth (Malcolm, 1979; Frisancho et al., 1983). In the Asai and Bundi tribes of New Guinea, for example, males continue to grow well into their twenties, while European males cease to grow at about 18 years of age (Malcolm, 1970). Roche and Davilla (1972) report the average age at completion of significant linear growth is 17.3 years in Caucasian U.S. girls.

Malcolm (1970) asserts that chronic protein and calorie malnutrition prolongs adolescent growth and development of Bundi children, although the onset of the adolescent spurt is not significantly affected. Furthermore, these data suggest that malnutrition compromises adult height. In contrast, a study of Kenyan adolescents suggests that the onset of puberty is delayed in undernourished children, while the progression of puberty is similar in well- and undernourished children (Kulin et al., 1982, 1984).

Menarche occurs relatively late in the development process, after the period of greatest acceleration in height, or peak height velocity (PHV), and when development of secondary sex characteristics is nearing completion. The mean age at PHV is about 12 years in Northern European girls and North American girls of Northern European extraction, while the mean age of menarche is between 12.7 and 13.2 years (Tanner, 1978). Menarche, on average, occurs 1.3 years after PHV with a range of $0-2.5$ years, and only $1 \%$ of girls reach menarche before PHV (Marshall and Tanner, 1986).

A relationship between menarche and nutritional status has been well established (Frisch, 1985; Tanner, 1962), although the exact mechanisms remain the subject of some controversy (Ellison, 1982; Trussell, 1978; Scott and Johnston, 1982). In the results presented below the differences in growth between pre- and postmenarcheal adolescents in Bangladesh are examined in some detail, and the findings are compared to studies of well-nourished adolescents.

\section{DATA AND METHODS}

Data were collected by the International Centre for Diarrhoeal Disease Research, Bangladesh (ICDDR,B). The ICDDR,B has maintained a Demographic Surveillance System of vital elements in the Matlab Thana region of Bangladesh since 1963. Periodic censuses have been taken since 1966. In the present survey, 13 villages were purposely selected for ease of access. There were 1,618 girls, aged 8-18 years (10-20 years in 1976 ), registered in the 13 Matlab villages in the census of 1974. Attempts were made to contact all of these girls, and 1,448 nonpregnant females were interviewed at least once between March of 1976 and February of 1977.

TABLE 1. Population of original sampling frame, exclusions and final study sample by age

\begin{tabular}{|c|c|c|c|c|c|c|c|c|c|}
\hline \multicolumn{3}{|c|}{ Original sample } & \multicolumn{3}{|c|}{ Exclusions } & \multicolumn{4}{|c|}{ Final sample } \\
\hline & Tot & & \multirow{2}{*}{$\begin{array}{c}\text { Never } \\
\text { measured } \\
(\mathrm{N})\end{array}$} & \multirow{2}{*}{$\begin{array}{c}1 \text { or } 2 \\
\text { measures } \\
(\mathrm{N})\end{array}$} & \multirow{2}{*}{$\begin{array}{l}\text { Other } \\
(\mathrm{N})^{1}\end{array}$} & \multirow{2}{*}{$\begin{array}{l}\text { Premenarche } \\
\text { (N) }\end{array}$} & \multirow{2}{*}{$\begin{array}{l}\text { Postmenarche } \\
(\mathrm{N})\end{array}$} & \multicolumn{2}{|c|}{ Total } \\
\hline Age (yr) & $\mathrm{N}$ & $(\%)$ & & & & & & $\mathrm{N}$ & (\%) \\
\hline 10.5 & 245 & (15) & 15 & 133 & 1 & 96 & - & 96 & (9) \\
\hline 11.5 & 174 & (11) & 8 & 23 & 2 & 141 & - & 141 & (13) \\
\hline 12.5 & 153 & (10) & 16 & 2 & 1 & 134 & - & 134 & (13) \\
\hline 13.5 & 170 & (11) & 10 & 9 & 4 & 145 & 2 & 147 & (14) \\
\hline 14.5 & 166 & (10) & 10 & 18 & 3 & 115 & 20 & 135 & (13) \\
\hline 15.5 & 135 & (8) & 11 & 11 & 3 & 72 & 38 & 110 & (10) \\
\hline 16.5 & 174 & (11) & 20 & 23 & 14 & 44 & 73 & 117 & (11) \\
\hline 17.5 & 126 & (9) & 21 & 23 & 20 & 15 & 47 & 62 & (6) \\
\hline 18.5 & 122 & (8) & 26 & 19 & 16 & 5 & 56 & 61 & (6) \\
\hline 19.5 & 69 & (4) & 14 & 12 & 7 & 6 & 30 & 36 & (3) \\
\hline 20.5 & 82 & (5) & 16 & 17 & 24 & 1 & 24 & 25 & (2) \\
\hline Total & $1,616^{3}$ & $(100)$ & 167 & 290 & 95 & $774^{2}$ & 290 & 1,064 & $(100)$ \\
\hline
\end{tabular}

${ }^{1}$ Includes pregnancies (34), missing data for key variable, and late entry into the study.

2118 of these began menstruating during the period of observation.

"Two subjects were excluded because of missing age data. 
Subjects were measured once a month for a one-year period. Not all subjects were available for every interview, and a minimum of three height and weight measurements were required for a subject to be included in this analysis. A total of 290 girls (15\% of those ever interviewed) were excluded from the analysis because they failed to meet this criterion.

Table 1 shows the reason for exclusion by age and summarizes the change in age structure that occurs under the study criteria. Since exclusion from the study is related to age, the age structure of the sample is altered by the pattern of missing data. The most important difference is the decrease in the proportion of subjects aged 17 years and above. In the original sampling frame, $25 \%$ of all subjects were 17 years or older, compared to $9 \%$ after design criteria and data cleaning disqualified 554 subjects. Subjects 18 years and older thus cannot be considered as a representative sample of girls of that age in the Matlab population. In addition, these subjects have fewer measurements taken over the one-year follow-up on average than do younger subjects, because dropout rates were higher for older subjects. Therefore, estimates of growth rates for subjects 18-20 years of age are less reliable than for younger subjects.

Interviews were conducted from March 1976 through February 1977 by local women field assistants. At the initial interview, retrospective information on socioeconomic status, pregnancy, marital status, and menarche status was collected. In addition, height in centimeters and weight in kilograms were measured in the girls' homes. Interviews were repeated at monthly intervals. Measurements were collected, and the girls were asked about their menstrual status at each interview. Ages were adjusted upward 10 years from the 1966 census. When the census data were collected, parents' reported age of child was compared with intervals between successive siblings to improve accuracy of age reporting. It is unlikely that any girls under age 10 are included in the sample, because registration of birth dates began in 1966 . The most probable incidence of age misreporting occurs among the 15-20-year-old girls. Unmarried girls, who were classified as 10-14 years in 1966, may have underreported their ages; thus, it is possible that some subjects listed as 15-20 in 1976 may in fact be older. Since ages are recorded as age at last birthday, the data were adjusted to represent the average age within each one-year interval.

Weight in kilograms is measured to the nearest $0.1 \mathrm{~kg}$, taken with bare feet and light clothing. Scales were balanced with premeasured weights before each weighing. Standing height is measured to the nearest $0.1 \mathrm{~cm}$ with a portable stadiometer. Static indicators of nutritional status are weight for age, height for age, and Quetelet index $\left(\mathrm{kg} / \mathrm{cm}^{2}\right)$, calculated from the first measurement of height and weight to avoid seasonal variation in measurements.

The U.S. National Center for Health Statistics (NCHS) growth standards (National Center for Health Statistics, 1977) were selected for the standard population, as recommended by the World Health Organization (World Health Organization, 1983). The use of standards for an adolescent population presents several difficulties, since normal children begin their growth spurt within a wide range of ages. Genetic differences and environmental factors, such as altitude and climate, may also influence the timing and magnitude of growth. These issues are raised frequently when U.S. standards are used to assess non-white or non-U.S. populations. While useful for in-country screening programs, national or regional standards for developing country populations have several disadvantages for assessing the overall level of nutritional status, or for making international comparisons. The high prevalence of undernutrition in these areas artificially lowers the definition of normal. Moreover, comparison studies of children from the general population and from a select group of well-off families in Haiti and Egypt, strongly suggest that well-nourished children from a variety of racial groups and environments have heights and weights very similar to those of U.S. children included in the NCHS standards. Children from lower socioeconomic strata, in comparison, fell well below the same standards (Department de Sante Publique et Population, Republic of Haiti, 1979; Nutrition Institute, Ministry of Health, Arab Republic of Egypt, 1979).

In addition to static measures of nutritional status, the rate of change in height and weight is examined. Estimates of growth in height and weight in one year are plotted by age to construct cross-sectional growth curves for the study population. These are compared to cross-sectional curves for Brit- 
ish children from the Harpenden Growth Study (Tanner et al., 1966) to study differences in the timing and shape of the curves. Differences in rates of growth by socioeconomic status and age at menarche are also examined.

This analysis combines cross-sectional and longitudinal data, whereby a range of ages is followed for a relatively short time period of one year. If all girls were measured at the same times, then yearly growth could be calculated by summing the monthly increments, or by predicting height and weight at time $x+1$ from height and weight at time $x$. However, only $44 \%$ of the sample has the same starting and ending points, and many subjects have randomly missing data between the first and last interviews. Using methods that require balanced data would necessitate that all subjects with missing data be dropped from the analysis, thereby reducing the sample size by over one half. An optimal estimate for the purpose of this research would provide accurate and comparable measures of the increase in height and weight that each subject experienced over the twelve months of follow-up, without a substantial reduction in size, or significantly altering the structure of the sample.

Ordinary least squares (OLS) regression of height and weight against time (12 months) was selected to estimate the rate of growth in one year. Although growth over the adolescent period is non-linear, a linear approach works reasonably well over a oneyear interval, and these estimates are similar to actual increases in height in one year in a sub-sample of subjects. For each subject, a separate regression of height and weight against time is performed and the slope estimates are used to approximate change over the one-year period. Since all the height and weight values taken from each person are included in the estimate, individual measurement errors are less influential, thus minimizing the random error component in each estimate. The OLS method also allows the sample size to remain large, and maintains the age structure and other characteristics of the original sample.

To validate the use of OLS as an accurate estimate of growth in one year, OLS estimates were compared with the total increase in height and weight in one year for a subgroup of 470 girls for whom 12 measurements were available, and to OLS estimates for the entire sample and the same subset

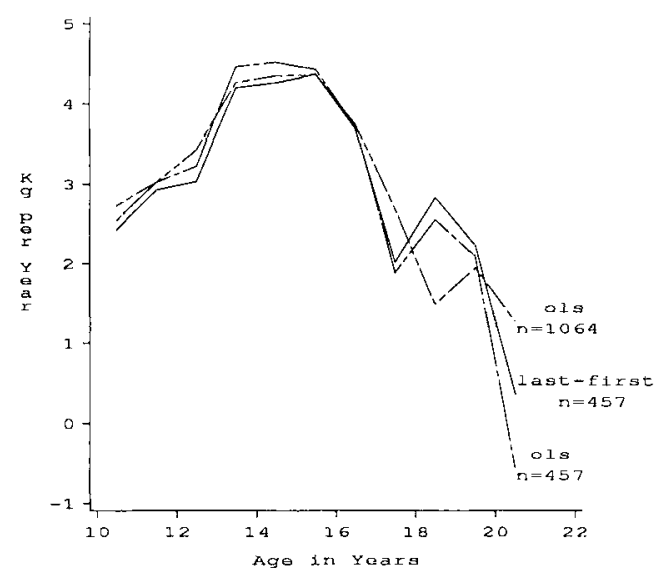

Fig. 1. Kilograms per year by age: OLS estimates for all subjects $(n=1,064)$ and OLS and total increments (last-first) for 457 women with no missing data.

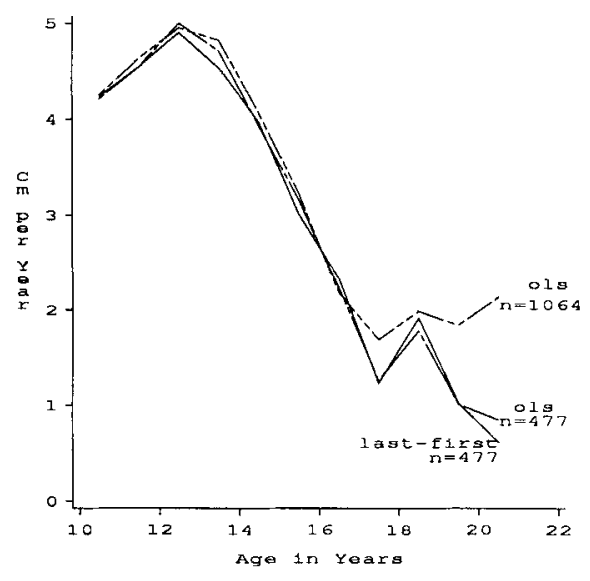

Fig. 2. Centimeters per year by age: OLS estimates for all subjects $(n=1,064)$ and OLS and total increments (last-first) for 477 women with no missing data.

(see Fig. 1,2). The OLS estimates and total increments are virtually identical in the subsample with 12 equidistant measurements. Likewise, OLS estimates for the total sample produce very similar estimates until about age 17 . However, the OLS method tends to overestimate growth in one year, particularly for height, in subjects age 18 years and older. The overestimate is attributed to two factors. Most important, growth rates decline with time after menarche. Secondly, seasonal patterns of growth are such that more growth occurs in the first few months of 
observation (e.g., March-April), especially for height. Subjects with less than five measurements tended to drop out after the first few interviews, thus concentrating their measurements in the period of most rapid growth for that year.

\section{RESULTS}

Percentile distributions of weight for age in the sample population are compared to the NCHS median in Figure 3. The median for U.S. girls is greater than the 95th percentile for Bangladeshi girls at every age, but the difference between the medians and the reference standard decreases as age increases, thus indicating that the growth spurt in weight is relatively late in Bangladeshi girls. A difference of $16.7 \mathrm{~kg}$ persists in the 20th year, although some catch-up growth appears to occur. Percentile distributions of height for age in Bangladeshi girls and the standard population are displayed in Figure 4. Girls in the study population are also short for their age compared to the NCHS standard. Delayed onset of the adolescent spurt in height and some catch-up growth are also suggested. The age at which growth is complete in this population is unknown, but it is unlikely that the Bangladeshi girls will ever attain heights that are similar to the standard. Huffman et al. (1985) shows that the heights of non-pregnant married Bangladeshi women are also low; mean height of women aged $20-29$ years is $148.3 \mathrm{~cm}$.

Mean weight for age and height for age by menarcheal status are compared in Figures 5 and 6 . Girls are categorized as postmenarcheal or premenarcheal at the beginning of the study period, and the first height and weight measurement on each subject (no later than month 3 of the study period) is used. Postmenarcheal girls are heavier than premenarcheal girls at every age (Fig. 5). In general, weight increases with age for both groups, although premenarcheal girls have the greatest increase in weight with age. Height for age by menarche status is shown in Figure 6. The trends are similar to those for weight, with postmenarcheal girls being taller than premenarcheal girls at every age. This is consistent with studies of well-nourished populations with early age at menarche (Moerman, 1982; Frisch and Revelle, 1970; Tanner, 1978).

Height and weight at menarche are examined separately for girls who attained menarche during the period of observation.

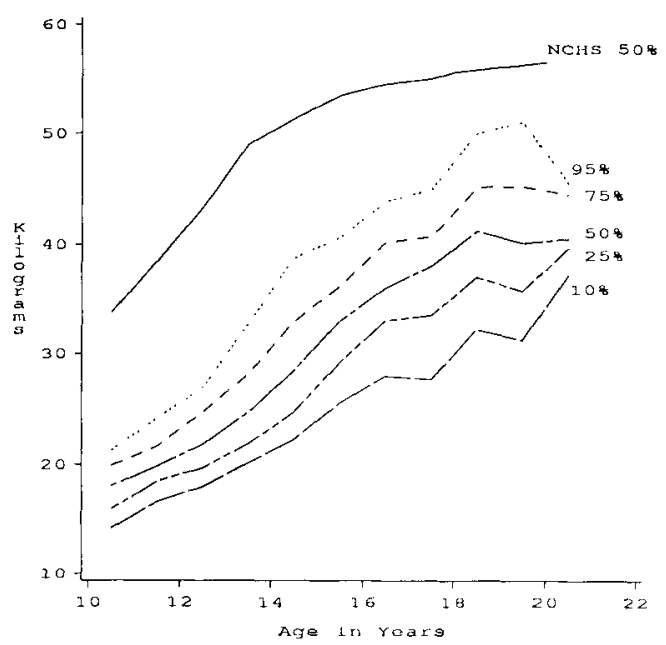

Fig. 3. Percentile distribution of weight for age in Bangladeshi females and NCHS median.

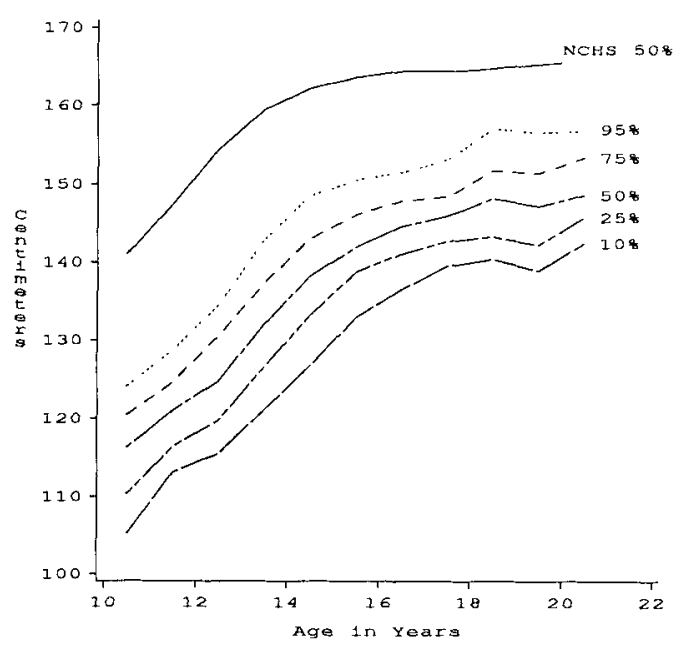

Fig. 4. Percentile distribution of height for age in Bangladeshi females and NCHS median.

Figure 7 shows mean weight at menarche and confidence intervals for 98 girls who had menarche during the study, and were measured in that month. Weight at menarche appears to increase with age until 17.5 years, and then decrease for the two oldest age groups. However, the sample size is small for girls less than 13.5 and greater than 16.5 years of age. There is a slight trend for the 13.5-16.5 group, but confidence intervals for 


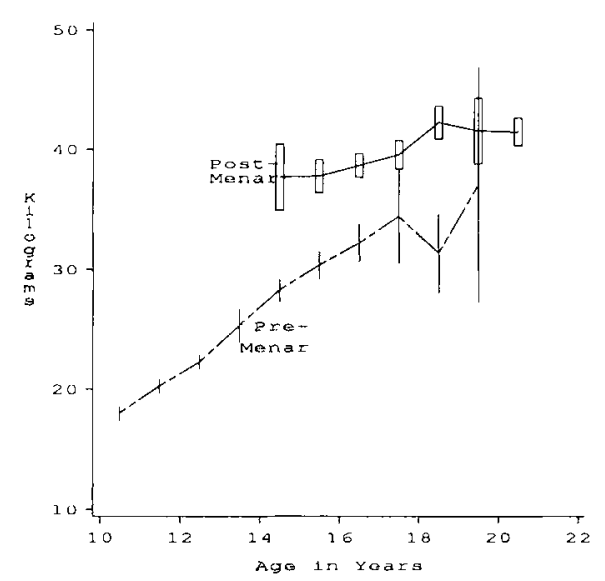

Fig. 5. Mean weight by age among pre- and postmenarcheal females. Note: Bars represent $95 \%$ confidence limits.

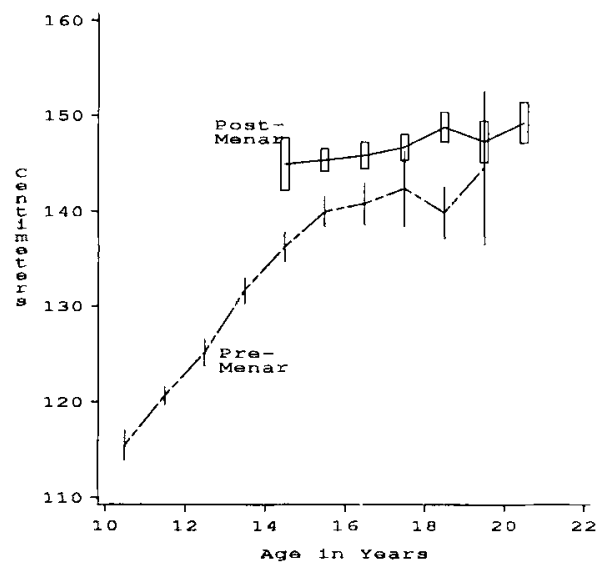

Fig. 6. Mean height by age among pre- and postmenarcheal females. Note: Bars represent $95 \%$ confidence limits.

the average weight overlap considerably at each age. Height at menarche follows a similar pattern (Fig. 8), although the increase in height with age at menarche is even less distinct than that for weight. The slight increasing trend in height and weight at menarche with age at menarche suggests that girls with late age at menarche have completed a greater proportion of their growth prior to menarche than have earlier maturing girls. Girls with age at menarche of 18.5 years and older appear to have lower weights and heights, possibly because both menar-

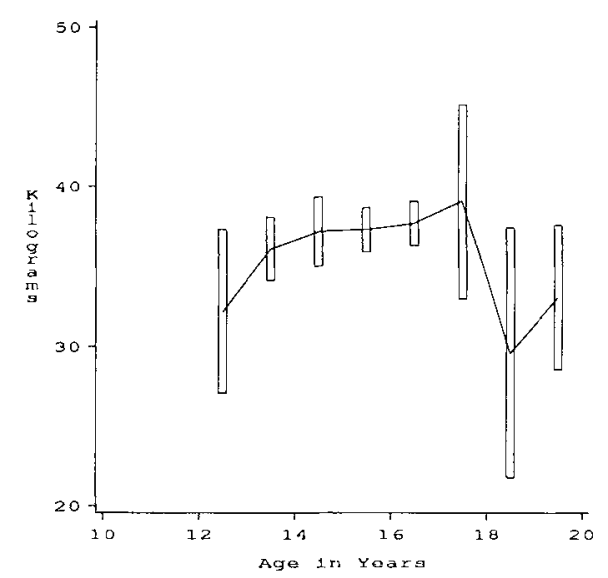

Fig. 7. Weight at menarche by age at menarche $(\mathrm{n}=98)$. Note: Bars represent $95 \%$ confidence limits.

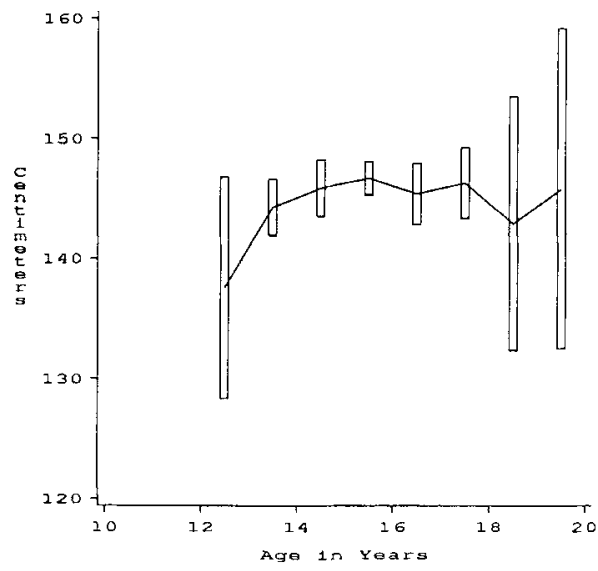

Fig. 8. Height at menarche by age at menarche ( $n=98$ ). Note: Bars represent $95 \%$ confidence limits.

che and growth were delayed by poor nutrition. However, sample sizes in the oldest three and youngest two age groups are too small to permit any conclusions, and the possibility of errors in age reporting cannot be ruled out.

In addition to low weight and height for age, Bangladeshi girls are relatively thin for their height compared to U.S. girls of the same age. Percentile distributions of the Quetelet index of the sample are compared to Health and Nutrition Examination Survey-1 (HANES-1) data for white females in Figure 


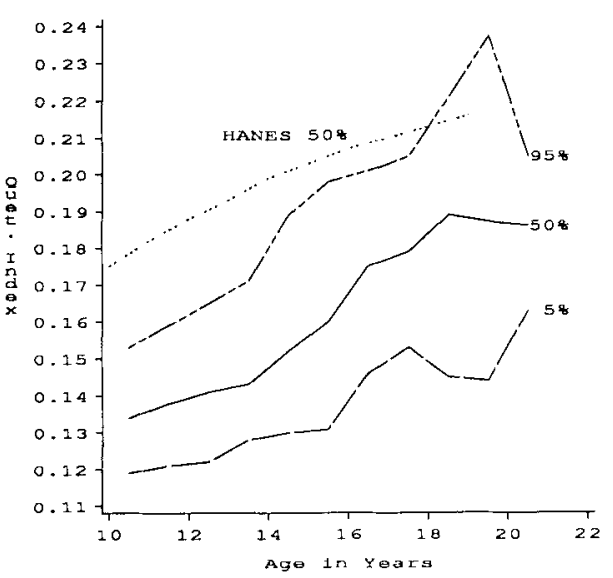

Fig. 9. Percentile distributions of Quetelet Index (kg/ $\left.\mathrm{cm}^{2}\right) \times 100$ by age for Bangladeshi girls compared to HANES median.

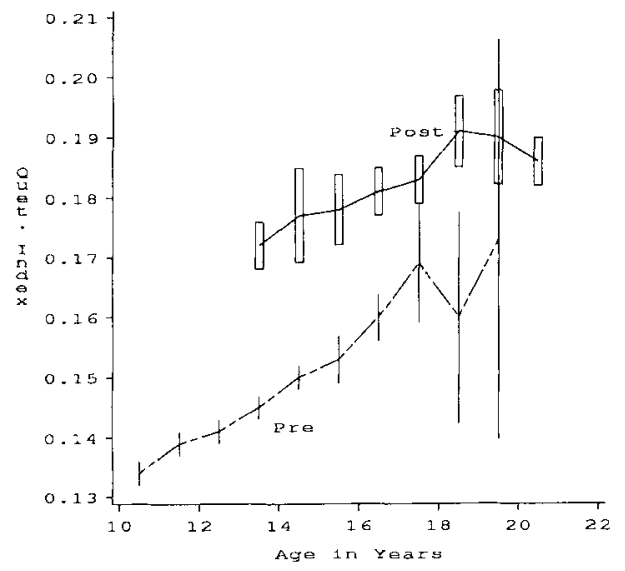

Fig. 10. Median Quetelet Index $\left(\mathrm{kg} / \mathrm{cm}^{2}\right) \times 100$ for pre- and postmenarcheal girls. Note: Bars represent 95\% confidence limits.

9 (Cronk and Roche, 1982). The median Quetelet index for the Bangladeshi girls is below the 5th percentile of the HANES-1 data until age 16.5 years, and the 95 percentile is less than the HANES-1 median until age 18.5. Weight for height increases more with age in the Bangladeshi girls. In the HANES-1 data, weight for height begins to level off after age 13 years, while Bangladeshi girls continue to increase in weight at a faster rate into the older age groups. Postmenarcheal girls are relatively heavier

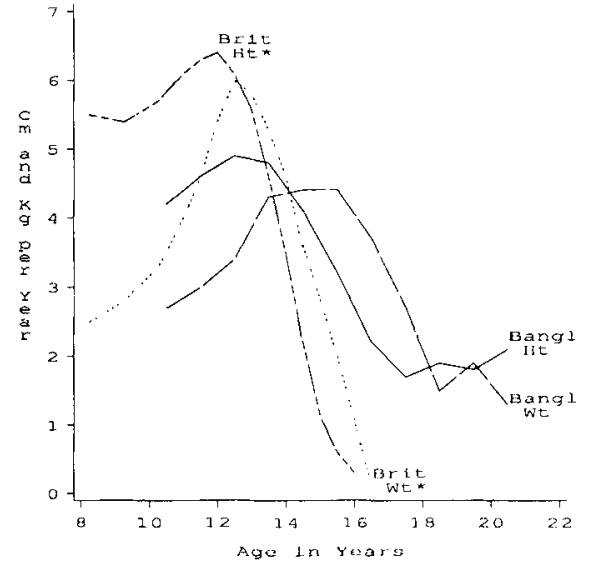

Fig. 11. Height and weight velocity by age for Bangladeshi and British adolescents. *Tanner et al. (1966).

than premenarcheal girls the same age at virtually every age (Fig. 10).

The entire growth curve is shifted forward in time for the Bangladeshi girls compared to standards for British adolescents (see Fig. 11). Although the onset of the growth spurt in the Bangladeshi adolescents cannot be identified, since the youngest girls are 10.5 years old on average at the beginning of the study, the adolescent spurt in both height and weight appears to begin as well as end later in Bangladeshi girls compared to British girls (Tanner et al., 1966). In addition, the growth spurt is less intense; the highest average rate of growth for Bangladeshi girls is $4.9 \mathrm{~cm}$ per year (at 12.5 years), compared to $6.4 \mathrm{~cm}$ per year (at 12.0 years) in the British standard. Height velocity reaches 8.3 $\mathrm{cm}$ per year in North American girls at 12 years (Tanner and Davies, 1985; data not shown). Standard curves developed for North American adolescents (Tanner and Davies, 1985) are similar to the British standard, although the peak in height velocity is somewhat greater and occurs slightly earlier (data not shown). It appears that menarche may also occur somewhat later in relation to PHV in Bangladeshi adolescents compared to British and U.S. adolescents. However, these data do not permit the determination of age at PHV on an individual basis; therefore, this finding is merely suggestive.

Average height and weight velocity per year for pre- and postmenarcheal girls is shown in Figures 12 and 13. Transitional 


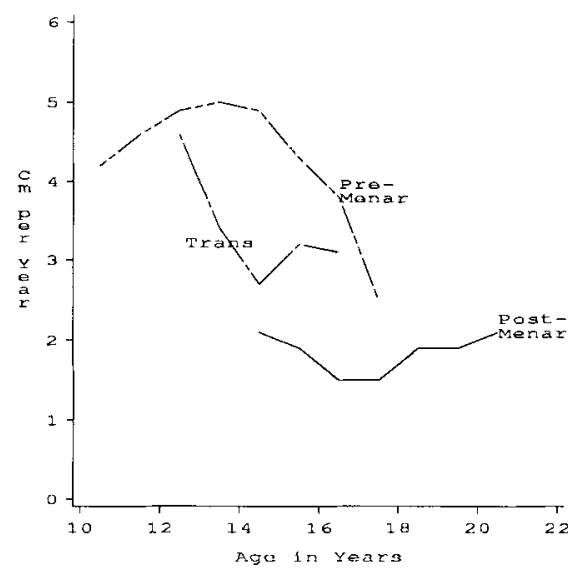

Fig. 12. Mean height velocity by age and menarche status.

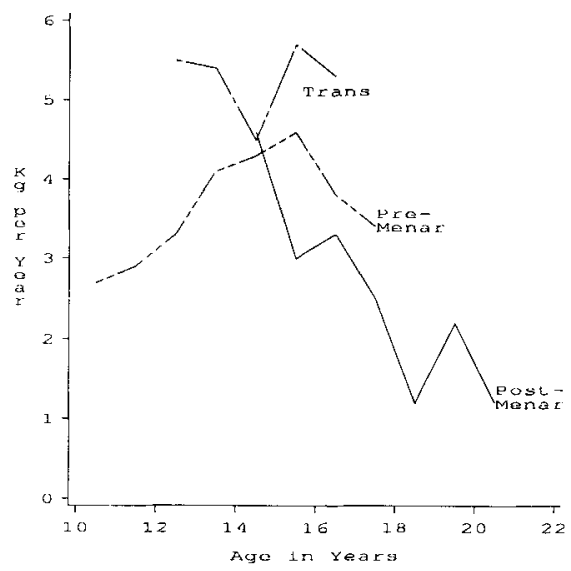

Fig. 13. Mean weight velocity by age and menarche status.

girls, those who reach menarche during the period of observation $(\mathrm{n}=118)$, are separated from postmenarcheal girls because of their proximity to PHV. Groups containing fewer than five cases are excluded in these graphs. Premenarcheal girls have the highest rate of growth in height at every age (Fig. 12). Girls who reach menarche during the period of observation are intermediate, and postmenarcheal girls have the lowest height velocity at almost every age. The pattern for weight gain per year is somewhat different, with the highest rates of growth occurring in the transitional group (Fig. 13). Premenarcheal girls are intermediate, and postmenarcheal girls have the smallest weight gain per year at every age. The difference in the rate of growth in height and weight is explained by the relative timing of the growth spurt in height and weight. Since the spurt in weight follows the spurt in height, rapid deposition of weight continues after the rate of change in height begins to decline. Therefore, premenarcheal girls, on average, are closer to their peak level of growth in stature than either transitional or postmenarcheal girls. Weight gain, on the other hand, is higher around the time that first menses occurs.

The probability of menstruating increases with age for premenarcheal girls; thus, part of the age trend reflects temporal proximity to menarche. The rate of growth in height begins to decline after age 14 years in these girls. This suggests that girls with later ages at menarche are farther past their peak height velocity than earlier maturing girls, or that they experience slower rates of growth throughout the adolescent spurt. However, there is no way to determine when any individual will actually begin menstruating, so the independent contributions of age and age at menarche cannot be separated. Likewise, in the postmenarcheal group, older girls will be farther past menarche on average than younger girls. The effects of age and time since menarche will therefore be confounded. Moreover, growth after menarche may depend on the age at which menarche occurs. The relationship of age and time since menarche to postmenarcheal growth in this population has been analyzed elsewhere (Riley et al., 1989).

The relationship of socioeconomic and cultural variables to growth and nutritional status was also examined (data not shown). Variables used were mother's education, size of dwelling, and religion (Hindu or Muslim). There are small but consistent differences in height and weight for age. Having some education and a larger house are associated with greater height and weight for age. Likewise, Muslim girls who comprise the dominant ethnic group are taller and heavier than minority Hindu girls of the same age. Menarche is also earlier on average among these same groups, which may explain the differences. There are no consistent trends in Quetelet index or in the rate of growth per year by any of these variables. 


\section{SUMMARY AND DISCUSSION}

Bangladeshi girls are shorter and lighter for their age than U.S. NCHS standards. The difference between the sample population and the standard decreases with age, suggesting that part of the differential is caused by a delay in the onset of the adolescent spurt in weight and height. Bangladeshi girls are also thinner on average at any given height than U.S. Caucasian girls from HANES-I. Postmenarcheal girls are taller and heavier than premenarcheal girls the same age. This is consistent with studies of adolescent girls in both well- and poorly nourished populations.

One-year growth rates in height and weight show marked differences in both the timing of the adolescent growth spurt and the shape of the curve between Bangladeshi adolescents and adolescents from Britain and the U.S. Bangladeshi girls have a longer growth spurt and a lower rate of growth at every time since PHV for both height and weight. Since the precise starting and ending points of the adolescent growth spurt are unknown in this sample, the area under the curves could not be compared.

These data demonstrate that the relationship of nutritional status to menarche is consistent with previous research in both developed and developing countries. Postmenarcheal girls are taller and heavier than premenarcheal girls of the same age, even when the age at menarche is considerably delayed. In addition, findings presented here suggest that menarche occurs after PHV, while the most rapid weight gain occurs around the time of menarche. However, growth in malnourished adolescent girls is delayed compared to that in well-nourished populations, evidenced by height and weight for age, and the rate of growth in both parameters.

Several factors in the sampling and study design limit the ability to generalize these findings. The severe famine that occurred in Bangladesh in 1974-1975 may affect the representative structure of the sample population. Although data collection did not begin until one year later, the impact of severe food deprivation, resultant changes in fertility and mortality, and the disruption of social structures may have differentially affected various segments of the population. Of particular concern is the possibility that the effects of acute famine on growth and adolescent development may have been more pro- nounced for specific age groups. For example, age at menarche may have been delayed for girls who were approaching first menses at the time of the famine, whereas less mature girls and those who were postmenarcheal before the famine may have been less affected.

The adolescent spurt in height and weight could also be differentially affected at different ages and stages of development. The girls in this study might reflect a period of rapid catch-up growth, following growth deficits that may have occurred during the famine. Moreover, younger girls might be more likely to recover from this type of insult than older girls. The impact of famine conditions may also vary by socioeconomic status. Poorer families may have been harder hit, with fewer landholdings and possessions to sell in response to higher food prices and lower household production, although no evidence for this is apparent from this analysis or in multivariate research on the factors affecting postmenarcheal growth (Riley et al., 1989).

Results from this study have important policy implications for nutrition programs in developing countries. They suggest a need to reconsider current strategies for nutrition education and intervention programs which primarily target infants and children, and pregnant and lactatirg women. These findings suggest that adolescent girls and nulliparous young women comprise another important high-risk group. Improving the nutritional status of adolescent girls and young women could positively influence both women's health and birth outcome, as these young women mature and move into their childbearing years. This is an especially important consideration in Bangladesh and other developing countries where women have very low social status. Young women, who have not yet begun childbearing, are often a very low priority in terms of food and other household resources.

\section{ACKNOWLEDGMENTS}

Data for this research were generously provided by the International Centre for $\mathrm{Di}$ arrhoeal Disease Research, Bangladesh. Sandra Huffman and A.K.M. Chowdhury collected the original data for this study in 1976-1977. The author gratefully acknowledges Drs. Huffman and W.H. Mosely for their valuable advice, and Barbara Zsembik for her comments on the manuscript. Thanks 
also to Judy Mullin for preparation of the manuscript. This research was supported in part by the Andrew W. Mellon Foundation.

\section{LITERATURE CITED}

Cronk CE, and Roche AF. (1982) Race- and sex-specific reference data for triceps and subscapular skinfolds and weight/stature. Am. J. Clin. Nutr. 35:347-354.

Department de Sante Publique et Population, Republic of Haiti (1979) Haiti Nutrition Status Survey: 1978. USAID.

Ellison PT (1982) Skeletal growth, fatness, and menarcheal age: A comparison of two hypotheses. Hum. Biol. 54(2):269-281

Eveleth PB, and Tanner JM. (1976) Worldwide Variation in Growth. London: Cambridge University Press.

Foster DL, Menken J, Chowdhury AKM, and Trussel J (1986) Female reproductive development: A hazards model analysis. Soc. Biol. 33(3-4):183-198.

Frisancho AR, Matos J, and Flegal P (1983) Maternal nutritional status and pregnancy outcome. Am. J. Clin. Nutr. 38:739-746.

Frisch RE (1972) Weight at menarche: Similarity for well-nourished and undernourished girls at different ages and evidence for historical constancy. Pediatrics $50(3): 445-450$.

Frisch KE (1985) Fatness, menarche, and female fertility. Perspect. Biol. Med. 28(4):611-633.

Frisch RE, and Revelle R (1970) Height and weight at menarche and a hypothesis of critical body weights and adolescent events. Science 16:397-398.

Huffman SL, Ford K, Allen HA, and Streble P (1985) Nutrition and fertility in Bangladesh: Nutritional status of nonpregnant women. Am. J. Clin. Nutr. 42: 725-738.

Kulin HE, Bwibo N, Mutie D, and Santner SJ (1982) The effects of chronic childhood malnutrition on pubertal growth and development. Am. J. Clin. Nutr. 36(3):527-536.

Kulin HE, Bwibo N, Mutie D and Santner SJ (1984) Gonadotropin secretions during puberty in malnourished children. J. Pediatr. 105(2):325-328.

Malcolm LA (1970) Growth and development of the bundi child of the New Guinea Highlands. Hum. Biol $42: 293-327$.
Malcolm LA (1979) Protein-energy malnutrition and growth. In J Falkner and JM Tanner (eds.) Human Growth, 3:361-372, First Edition. New York: Plenum Press.

Marshall A and Tanner JM (1979) Puberty. In J Falkner and JM Tanner (eds.) Human Growth, 2:171-210, First Edition. New York: Plenum Press.

Marshall A, and Tanner JM (1986) Puberty In J Falkner and JM Tanner (eds.) Human Growth, 2:171-209, Second Edition, New York: Plenum Press.

Moerman ML (1982) Growth of the birth canal in adolescent girls. Obstet. Gynecol. 143:528-532.

National Center for Health Statistics (NCHS) (1977) NCHS Growth Curves for Children, Birth-18 Years: United States. Series 11(167), Hyattsville, Maryland.

Nutrition Institute, Ministry of Health, Arab Republic of Egypt (1979) Arab Republic of Egypt Nutrition Status Survey: 1978. USAID.

Riley AP, Huffman SL, and AKM Chowdhury (1989) Age at menarche and postmenarcheal growth among rural Bangladeshi females. Ann. Hum. Biol. 16(4):347359 .

Roche AF, and Davilla GH (1972) Late adolescent growth in stature. Pediatrics 50(6).

Scott EC, and Johnston FE (1982) Critical fat, menarche, and the maintenance of menstrual cycles: A critical review. J. Adolesc. Health Care 2:249-260.

Tanner JM (1962) Growth at Adolescence, 2nd ed., Oxford: Blackwell

Tanner JM (1978) Fetus Into Man. Cambridge, MA: Harvard University Press.

Tanner JM and Davies PS (1985) Clinical longitudinal standards for height and height velocity for North American children. J. Pediatr. 107(3):317-329.

Tanner JM, Whitehouse RH and Takaishi M (1966) Standards from birth to maturity for height, weight, height velocity and weight velocity: British children, 1965, Part II. Arch. Dis. Child. 41:613-635.

Trussell J (1978) Menarche and fatness: A re-examination of the critical body composition hypothesis. Science 200:1506-1509.

World Health Organization (1983) Measuring Change in Nutritional Status. WHO, Geneva.

Wyshak G (1983) Age at menarche and unsuccessful pregnancy outcome. Ann. Hum. Biol. 10(1):69-73. 